\title{
Analysis and Investigation of the Inverter for Energy Transfer from Small Wind Power Plant to Common Grid
}

\author{
Romuald Luczkowski and Roman Muszynski \\ Poznan University of Technology \\ Poland
}

\section{Introduction}

There are many different solutions of wind power systems sized for different power values (El-Tamaly et al.). The examples of the low power systems are shown in Fig.1. In the A structure (Chikaraishi et al., 1990) the induction generator along with the fully controlled rectifier is used. In the B system (Higuchi et al., 2000) a synchronous generator, diode rectifier and pulse converter for DC voltage control are applied. In the C system (Tyc, 2003; Muszynski \& Pilacinski, 2006) the energy is obtained using the large vehicle alternator.

The last solution can be considered in the plant of the lowest power range as the competitive one regarding two previous systems. It is foreseen as a non-industrial application for coupling the amateur-made plants with one-phase common grid.

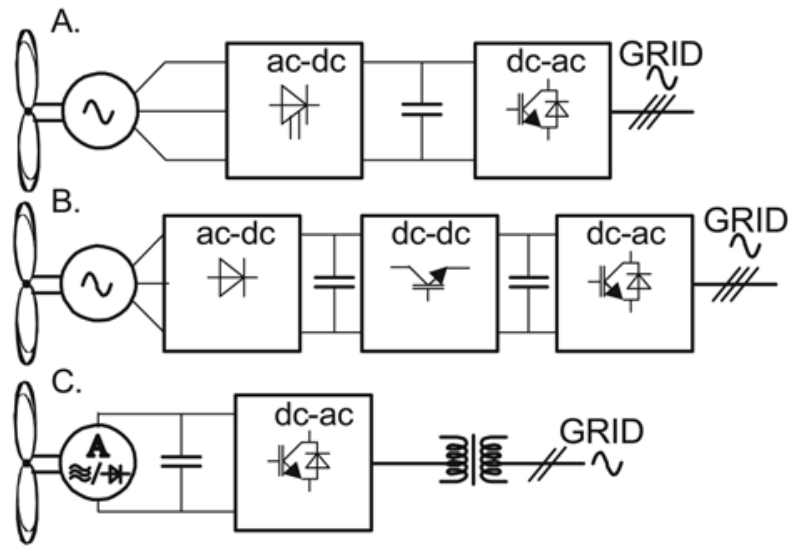

Fig. 1. The small wind energy systems

Need of "home-built" small wind power plants in many countries (Piggott, 2005) is motivation for the problem investigation. Such the plants of power one-to-several $\mathrm{kW}$ are built on the base of low speed permanent magnet synchronous machines with axial flux or on the base of car-, bus- or truck-alternators. Operation of these plants with accumulator bank only does not satisfy enthusiasts of own renewable energy. People would like to have

Source: Wind Power, Book edited by: S. M. Muyeen,

ISBN 978-953-7619-81-7, pp. 558, June 2010, INTECH, Croatia, downloaded from SCIYO.COM 
higher financial effect that will be reached if the produced energy can be transferred to the public network. There are not industrial inverters of such small power on the market. A trial of solution of this problem on the base of voltage source inverter with hysteresis current controller was undertaken.

In the chapter the problems of transfer of energy from small wind plant to the electrical network are studied. Firstly, the investigated system is described (Section2). Then, the theoretical and simulation analysis of operation of the system is given (Section 3), where the physical phenomena as well as problems related to its operation and design are widely described. Next the first laboratory plant is presented (Section 4). At the end, the original, cost and reliability oriented design of the converter is provided (Section 5).

\section{The investigated system}

The scheme of the considered wind power system is shown in Fig.2 (solution C in Fig.1). The system (Tyc, 2003; Muszynski \& Pilacinski, 2006) consists of the wind turbine, alternator, RC filter, voltage source inverter, VSI, and matching transformer, TR. The transformer gives not only the voltage adjustment of the source to the grid but is necessary also from point of view of safety. As persons without suitable conscience of electric shock can approach the equipment of small wind power plant by, it must be separated from the grid voltage.

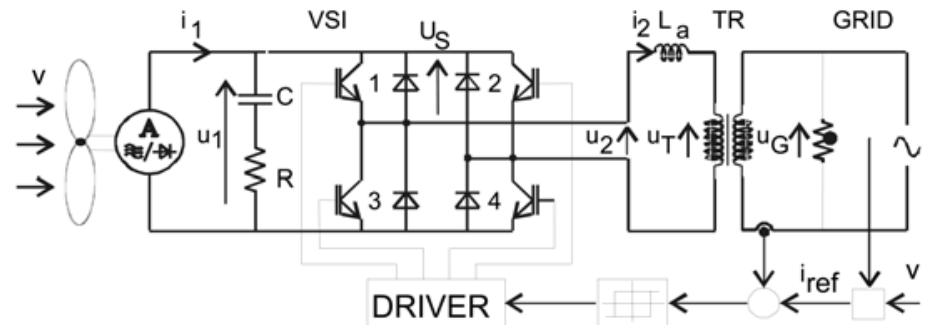

Fig. 2. Block diagram of the investigated system

The system from Fig.2 can not operate without filter between the source and load circuits. Direct switching both the circuits generates over-voltage of infinite value due to different initial values of currents flowing in the branches with inductances. Filter softens the switching phenomena.

It turns out (Section 3.4) that, for decreasing of the current slope rate and switching frequency of the inverter, the additional inductance into the output circuit should be inserted.

In the system the hysteresis-based output current control has been implemented. To obtain the unity power factor, the phase of current reference signal $i_{\text {ref }}$ should be opposite regarding to the phase of the network voltage. To provide the operation at the maximum power, the amplitude of signal should be adequate to wind velocity $v$.

The structure of the system (Fig.2) is the same as the structure used in the variable speed induction motor drive with inverter and hysteresis current controller (Mohan et al., 2003). However, there is fundamental difference in regard of parameter proportion between both the systems. It changes cardinal the conditions of design and operation of the system for transfer energy from small power plant to the common grid, in comparison with drive system where the energy flows from source of high power to receiver of smaller power. 


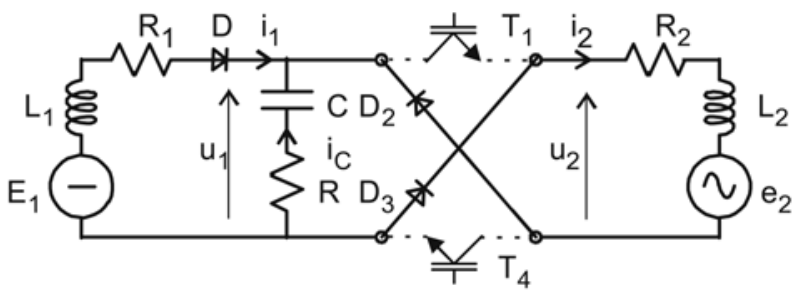

Fig. 3. Circuit configuration during D2 and D3 conducting (solid) as well as during transistor T1 and T2 conducting (dashed line)

The system: Small power source - Inverter with hysteresis current controller - Common grid from the Fig. 2 and 3 can be characterized as follows:

- $\quad$ The voltage (EMF) $E_{1}$ of the source (alternator) is small.

- The short circuit inductance $L_{1}$ of the source is great.

- The requested value of filter capacitance $C$ is great, in order to decrease voltage fluctuation.

- The frequency of the resonance in the circuit $L_{1} C$ is low.

- The natural inductance $L_{2}$ of the output circuit is small.

- The natural current slope rate and frequency of the hysteresis controller operation are high.

Because of above properties and values of the parameters the conditions of design and operation of the examined system are significantly more difficult than for the converter drive supplied from the grid. Damping of the resonance oscillation in the input circuit requires additional filter resistance. Decreasing of the current slope rate and frequency of the inverter operation requires additional choke in the output circuit. All the additional elements influence the power loss as well as the reliability and cost of the system.

The properties of the system as well as its operation and specific phenomena were studied by means of theoretical analysis and simulation investigation.

The following basic data and parameters of the system were taken into account:

- alternator: $E_{1}=28 \mathrm{~V}, L_{1}=0.175 \mathrm{mH}$,

- $\quad$ amplitude of the grid voltage $U_{G m}=311 \mathrm{~V}$,

- $\quad$ grid with transformer (voltage ratio $n_{T}=18.3$ ) reduced to the low voltage side:

- $\quad E_{2 m}=17 \mathrm{~V}, L_{T}=0.058 \mathrm{mH}, R_{2}=2.7 \mathrm{~m} \Omega$,

- additional inductance in output circuit: $L_{a}=0.08 \mathrm{mH}$,

- $\quad$ resistance of the additional choke $R_{a}=0$,

- total inductance of the output circuit $L_{2}=L_{T}+L_{a}=0,138 \mathrm{mH}$

- amplitude of sinusoidal reference current: $I_{2 m}=50 \mathrm{~A}$,

- hysteresis band: $\Delta i=6 \mathrm{~A}$,

- filter: $C=1 \mathrm{mF}, R=0.4 \Omega$.

In the chapter are given values of only those parameters, which differ from the above basic data. Transistors and diodes of the converter are assumed to be ideal (zero voltage drop and zero turn-off as well as turn-on time).

\section{Theoretical analysis and simulation of operation of the system}

\subsection{Operation of the system with basic parameters}

The figures 4, 5, 6 and 7 present one set of the quantities registered during simulating the system for basic parameters that give stable but not optimal operation of the converter. 


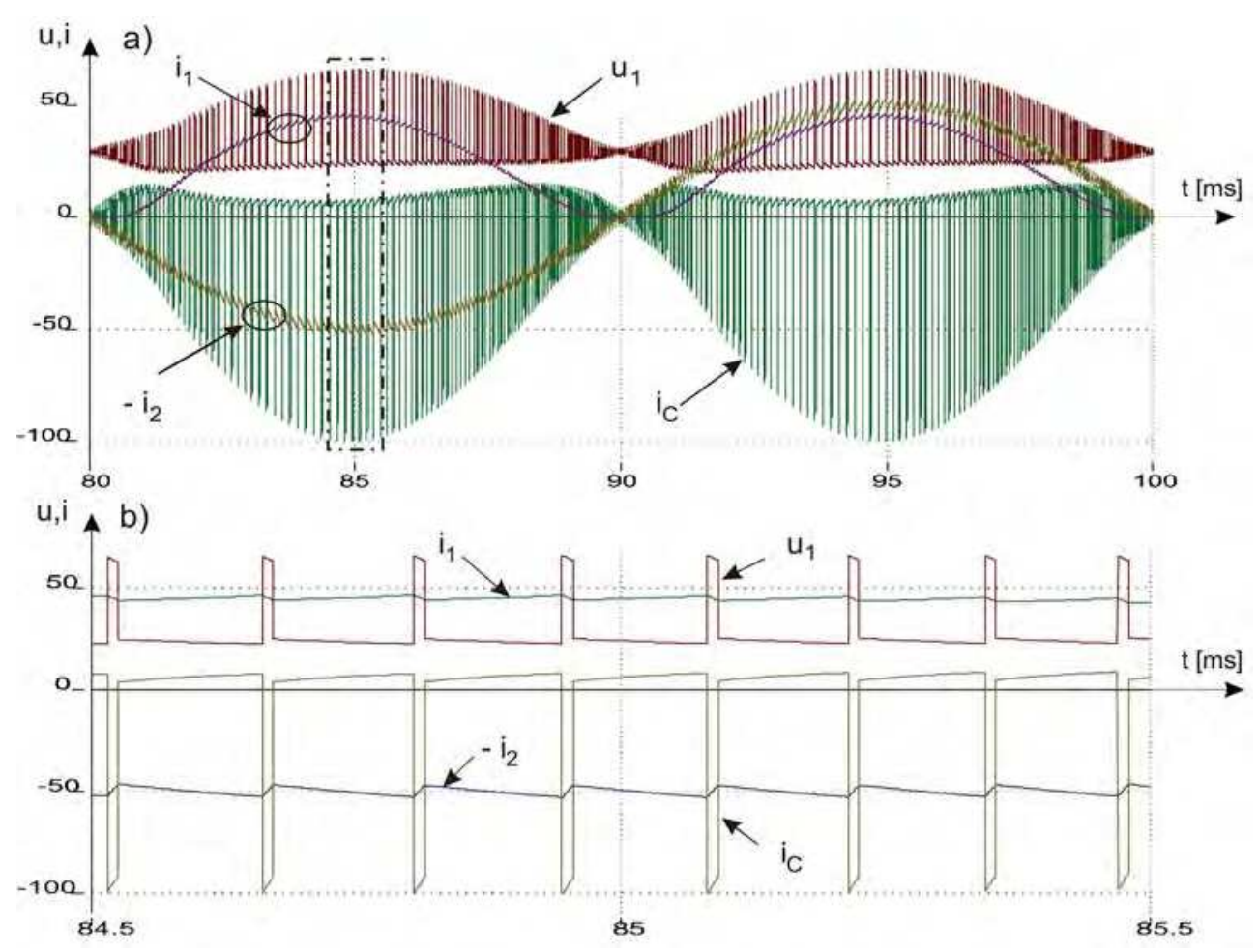

Fig. 4 . The course of filter voltage $u_{1}$ and current $i_{C}$ as well as current of source $i_{1}$ and receiver $i_{2}$ (Fig.a) and time extension of the course in region of current maximum (Fig.b) for basic data

It is seen from Fig.4a that the receiver current $i_{2}$ is sinusoidal. It follows the reference current $i_{\text {ref }} n_{T}$ with the error not greater than hysteresis band. The shape of the source (alternator) current $i_{1}$ (Fig.4a) complies in approximation with the rectified receiver current $i_{2}$. It can be also recognized as the near sin function of twofold frequency with DC component equal to half of its amplitude. The high frequency ripple of the current $i_{1}$ due to hysteresis controller operation is smaller than the receiver current one. Precise relation between the currents shown in Fig. 4 is

$$
i_{2}=i_{1}+i_{C}
$$

during transistor conducting and

$$
-i_{2}=i_{1}+i_{C}
$$

during diode conducting, according to Fig.3. The change of sign in the equations is in accordance with the switching the circuit configuration.

The course of $i_{C}$ in Fig.4 consists of the long positive-going pulses of small value (transistor on state) and the very short negative-going ones of very high value (diode conducting). The height of the negative pulses equals in approximation to the twofold absolute instantaneous 
value of the reference current. It can be substantiated as follow. At the end of transistor conducting $i_{C} \approx 0$ and according to (1) is

$$
i_{2}=i_{1}=i_{r e f} n_{T}
$$

In the transistor switch off state (diode switch on state) two currents are flowing into the filter: source current $i_{1}$ forced by inductance $L_{1}$ and current $i_{2}$ forced by inductance $L_{2}$. Then according to (2) is

$$
i_{C}=-\left(i_{1}+i_{2}\right) \approx-2 i_{r e f} n_{T} .
$$

The filter voltage $u_{1}$ is an important quantity in the system, as it appears on terminals of alternator, transistors, diodes and output circuit in accordance with states of the switches. It can be obtained using equations for the input circuit of the converter. For the alternator branch the following equation is valid:

$$
u_{1}=E_{1}-L_{1} \frac{d i_{1}}{d t}-i_{1} R_{1}-u_{D}
$$

which for the period of transistor conducting, can be simplified to the form:

$$
u_{1} \approx E_{1} \text {, }
$$

as $u_{D} \approx 0$ was assumed and, moreover, voltages on resistance $R_{1}$ and inductance $L_{1}$ are negligible due to small values of the resistance and current derivative. For the filter branch is:

$$
u_{1}=u_{C}-i_{C} R
$$

Due to small value of $i_{C}$ during transistor conducting, the formula (7) takes form:

$$
u_{1} \approx u_{C}
$$

Therefore, according to (6) and (8), during transistor conducting the filter voltage $u_{1}$ and capacitor voltage $u_{C}$ have approximately the same values equal to EMF $E_{1}$ of the source. During diode conducting the equation (7) takes the form

$$
u_{1} \approx E_{1}-i_{C} R=E_{1}+2 i_{2} R=E+2 i_{r e f} n_{T} R .
$$

The commutation overvoltage can be obtained in the form:

$$
r=\frac{u_{1}}{E_{1}}=1+2 \frac{i_{2} R}{E_{1}}
$$

The highest value of $u_{1}$ is reached in the region of current maximum. Taking into consideration, $I_{C m}=2 I_{r e f m} n_{T}=250 \mathrm{~A}$, result is $u_{m} \approx 28+100 \cdot 0,4=68 \mathrm{~V}$ for the data of the simulated plant. This value is visible in Fig.4.

Figure 5 shows the voltages of the system on the output side. The output voltage $u_{2}$ of the inverter equals to the filter voltage $u_{1}$ during transistor on state and $-u_{1}$ for transistor off state. The grid side voltage $u_{G}$ of the transformer is sinusoidal. Its amplitude equals to $311 \mathrm{~V}$ 


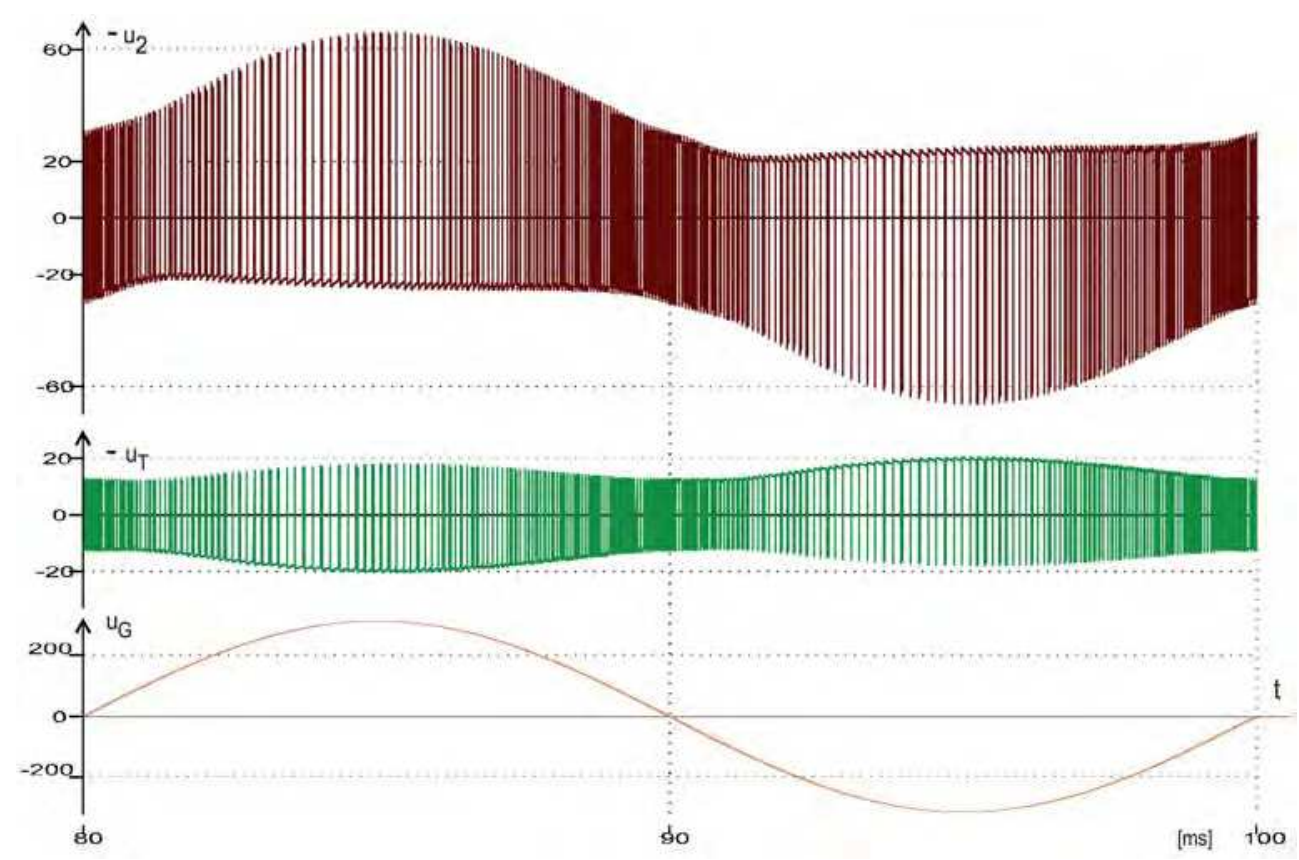

Fig. 5 . The course of the output, $u_{2}$, transformer, $u_{T}$, and grid, $u_{G}$, voltages for basic data and phase is opposite regarding to the phase of the reference current $i_{r e f}$. The shape of the inverter side voltage $u_{T}$ of the transformer is intermediate between the shape of $u_{2}$ and sinusoidal one, according to the equations:

$$
u_{T}=u_{2}-L_{a} \frac{d i_{2}}{d t}
$$

and

$$
u_{T}=-\frac{u_{G}}{n_{T}}+i_{2} R_{2}+L_{T} \frac{d i_{2}}{d t}
$$

where the negative sign at the voltage $u_{G}$ denotes the phase change between both the sides of the transformer. If the small value of the transformer resistance $R_{2}$ can be neglected then on the base of (11) and (12) is:

$$
u_{T} \approx \frac{L_{T}}{L_{a}+L_{T}} u_{2}-\frac{L_{a}}{L_{a}+L_{T}} \frac{u_{G}}{n_{T}} .
$$

For the simulated system is $L_{T}=0.058 \mathrm{mH}, L_{a}=0.08 \mathrm{mH}$ and according to these data the shape of $u_{T}$ in Fig.5 is influenced by $u_{2}$ with the weight of $42 \%$ and by $u_{G} / n_{T}$ with the weight of $58 \%$. It must be taken into account that in formulas (12) and (13) the phase of voltage $u_{T}$ is opposite in relation to the phase of the voltage $u_{G}$. The additional inductance $L_{a}$ is not profitable from the voltage point of view as it produces voltage drop 


$$
\Delta u_{a}=u_{2}-u_{T} \approx \frac{L_{a}}{L_{a}+L_{T}}\left(u_{2}+\frac{u_{G}}{n_{T}}\right) .
$$

It deteriorates the voltage matching of source (alternator) to the receiver (grid). The additional inductance $L_{a}$ is introduced to the system in order to decrease current slope rate and operation frequency of the inverter. This problem is separately analysed in Section 3.4. In efficiency analysis the power loss in the choke must be also taken into consideration, as there is not choke without resistance in the real system.

The high frequency voltage impulses $u_{T}$ presented in Fig.5 induce also additional loss in the magnetic circuit of the transformer, which is not taken into consideration in the presented model.

The course of the transistor (and diode) voltage is shown in Fig.6. Impulses of $u_{s}$ are the fragments of the voltage $u_{1}$ from Fig.4a, referring to the transistor off state. Hence, the maximum value of switch voltage can be obtained using formula (9) and maximum value of current $I_{2 m}$ or $I_{\text {refm }}$.

The power transferred between the separate elements of the system is registered in Fig.7. It is calculated as a simply product of voltage and current without any farther processing of the signals. In result of it the power registration in Fig.7 contains the impulse according to the course of current and voltage in Fig.4 and 5. Especially great and fast changes of the power take place on the inverter side terminals of the transformer $\left(p_{T}\right)$. The differences between the power in the separate points of the system, visible in the Fig.7, arise due to loss in the filter resistance (difference $p_{1}-p_{T}$ ) and - in the transformer resistance (difference $p_{T}$ $\left.p_{G}\right)$. The inverter as well as the additional choke is assumed for the simulation as the lossless element.

\subsection{The case of filter with reduced resistance}

In order to reduce the converter switching overvoltage given by formula (10) and loss in the filter, decrease of the filter resistance is analysed.

Lack of the resistance in the filter is a case of its extremely reduction. The filter voltage during converter switching can be obtained on the base of circuit equations, as the formula (11) is not valid for the case without resistance.

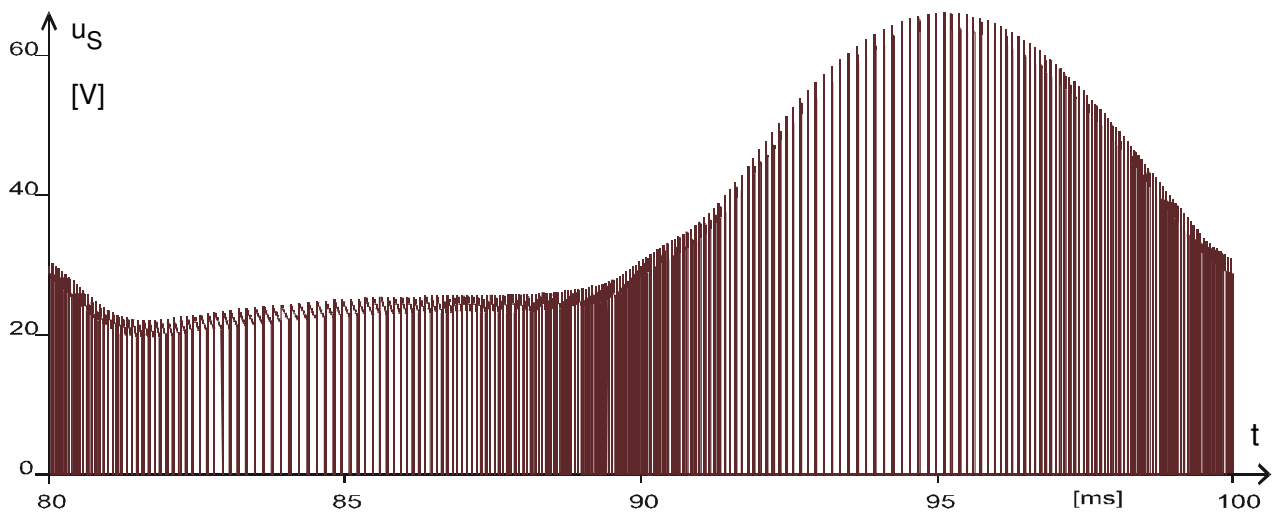

Fig. 6. The course of switch module voltage $u_{S}$ for basic data 


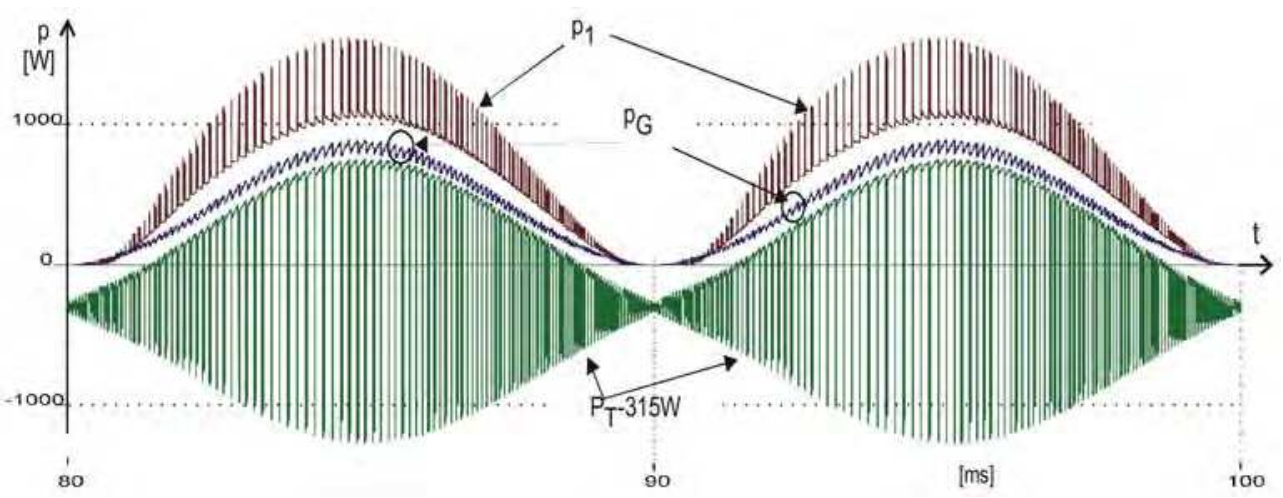

Fig. 7. The course of power on terminal of alternator, $\mathrm{p}_{1}$, transformer, $p_{T}$, and grid, $p_{G}$, for basic data

If the transistors are switching-off then the diodes are starting with conducting and the current $i_{2}$ flowing in the branch with output inductance decreases rapidly. In result of it the energy accumulated in the inductance decreases as well. Figure 3 shows configuration of the circuits at this stage. The following equations describe dynamical state:

$$
\begin{gathered}
L_{1} \frac{d i_{1}}{d t}=E_{1}-u_{1} \\
L_{2} \frac{d i_{2}}{d t}=-\left(u_{1}+e_{2}\right) .
\end{gathered}
$$

The rate of change of current $i_{2}$ on the base of (15) and (16) is:

$$
\frac{d i_{2}}{d t}=-\frac{E_{1}+e_{2}}{L_{2}}+\frac{L_{1}}{L_{2}} \cdot \frac{d i_{1}}{d t}
$$

It is seen that the derivative of $i_{2}$ is great, as the EMFs $E_{1}$ and $e_{2}$ act in the same direction. At this $e_{2}$ has a great value equal to the amplitude $E_{2 m}$ ( $i_{2}$ and $e_{2}$ are in the phase opposition). On the contrary, the rate of change of current $i_{1}$ is small, as on the right side of equation (15) is negative difference of two values, which are approached one to other.

In the state of system shown in Fig.3 the source 1 supplies the circuits with energy $\int E_{1} i_{1} d t$ and the grid receives energy $\int e_{2} i_{2} d t$. The initial conditions of the state and the small changes of currents and voltages (due to short duration of the state) allow assuming $E_{1} \approx e_{2}$ and $i_{1} \approx i_{2}$ in the above integrals. Therefore, it can be stated that these small energies of source and receiver balance one with other. In result of it, inner balance of energy in the converter circuits consists in its flowing from choke $L_{2}$ to the capacitor $C$. Decrease of choke current $i_{2}$ results in increase of filter voltage $u_{1}$. If the current $i_{2}$ decreases by hysteresis band $\Delta i$ then the energy accumulated in inductance $L_{2}$ decreases by the value:

$$
\Delta E=\frac{L_{2} I_{2 m}^{2}}{2}-\frac{L_{2}\left(I_{2 m}-\Delta i\right)^{2}}{2}=L_{2}\left(I_{2 m} \cdot \Delta i-\frac{\Delta^{2} i}{2}\right),
$$


where $I_{2 m}$ is the value of current before switching-off the transistors. The amplitude of output current of the converter is taken in the formula in order to obtain the maximum voltage increase. The energy flows to the capacitor. The increase $\Delta u$ of capacitor voltage $u_{1}$ is bounded with the energy according to the equation:

$$
\Delta E=C \frac{\left(u_{1}-\Delta u\right)^{2}}{2}-C \frac{u_{1}^{2}}{2}=C\left(u_{1} \cdot \Delta u+\frac{\Delta^{2} u}{2}\right) .
$$

Comparison of (18) and (19) gives the equation:

$$
L_{2}\left(I_{2 m} \cdot \Delta i-\frac{\Delta^{2} i}{2}\right)=C\left(u_{1} \cdot \Delta u+\frac{\Delta^{2} u}{2}\right) .
$$

The increase of capacitor voltage calculated from (20) is:

$$
\Delta u=\sqrt{u_{1}^{2}+2 \frac{L_{2}}{C}\left(I_{2 m} \cdot \Delta i-\frac{\Delta^{2} i}{2}\right)}-u_{1} .
$$

At the and of the transistor off state the filter, transistor, source and receiver voltage is:

$$
U_{m}=u_{1}+\Delta u=\sqrt{u_{1}^{2}+2 \frac{L_{2}}{C}\left(I_{2 m} \cdot \Delta i-\frac{\Delta^{2} i}{2}\right)} .
$$

For the initial value of $u_{1} \approx E_{1}$ the overvoltage written like (10) is

$$
r=\frac{U_{1 m}}{E_{1}}=\sqrt{1+2 \frac{L_{2}}{E_{1}^{2} C}\left(I_{2 m} \cdot \Delta i-\frac{\Delta^{2} i}{2}\right)} .
$$

In formulas (18) and (19) the terms $\Delta^{2} i / 2$ and $\Delta^{2} u / 2$ can be neglected. In this case instead of (20) is

$$
L_{2} I_{2 m} \cdot \Delta i=C u_{1} \cdot \Delta u
$$

and instead of $(21)$ is

$$
\Delta u=\frac{L_{2} I_{2 m} \cdot \Delta i}{C u_{1}}
$$

Filter containing capacitor without serial resistance is a good solution from point of view of softening switching phenomena. In this case the voltage jump $\Delta u$ during diode conducting is small. For $R=0$ and for the basic data of remaining parameters the jump according to (21) is $\Delta u=1,38 \mathrm{~V}$,only, and according to (25) is $\Delta u=1,5 \mathrm{~V}$.

Unfortunately, the operation without filter resistance can be inadmissible. Decrease of the resistance results in decrease of the overvoltage according to the formula (10) and of the power loss. The decreased voltage drop on filter resistance is visible in Fig. 8 in comparison with Fig.4a. But the resistance can not be too small due to resonance phenomenon.

The resonance arises in the circuit containing filter capacitance $C$ and source inductance $L_{1}$. It produces oscillations of voltage supplying the converter. Hysteresis shaping of the current 
separates efficaciously output circuit from the capacitor. In these conditions, inductance $L_{2}$ of the circuit does not influence resonance. Frequency of the oscillation is calculated now according to formula:

$$
f=\frac{1}{2 \pi \sqrt{L_{1} C}}
$$

and can be only a few times greater than the network voltage frequency. Amplitude of oscillation can be so great, that the voltage $u_{1}$ becomes in some periods of time much less than the receiver EMF $e_{2}$. It disturbs significantly the process of current forming and is unacceptable.

Such the situation is shown in Fig.9b. For the data of the simulated system the frequency is $f=381 \mathrm{~Hz}$ according to (26). It can be confirmed in Fig. $8 \mathrm{~b}$. According to the oscillating value of $u_{1}$ the switching frequency changes in a wide range.

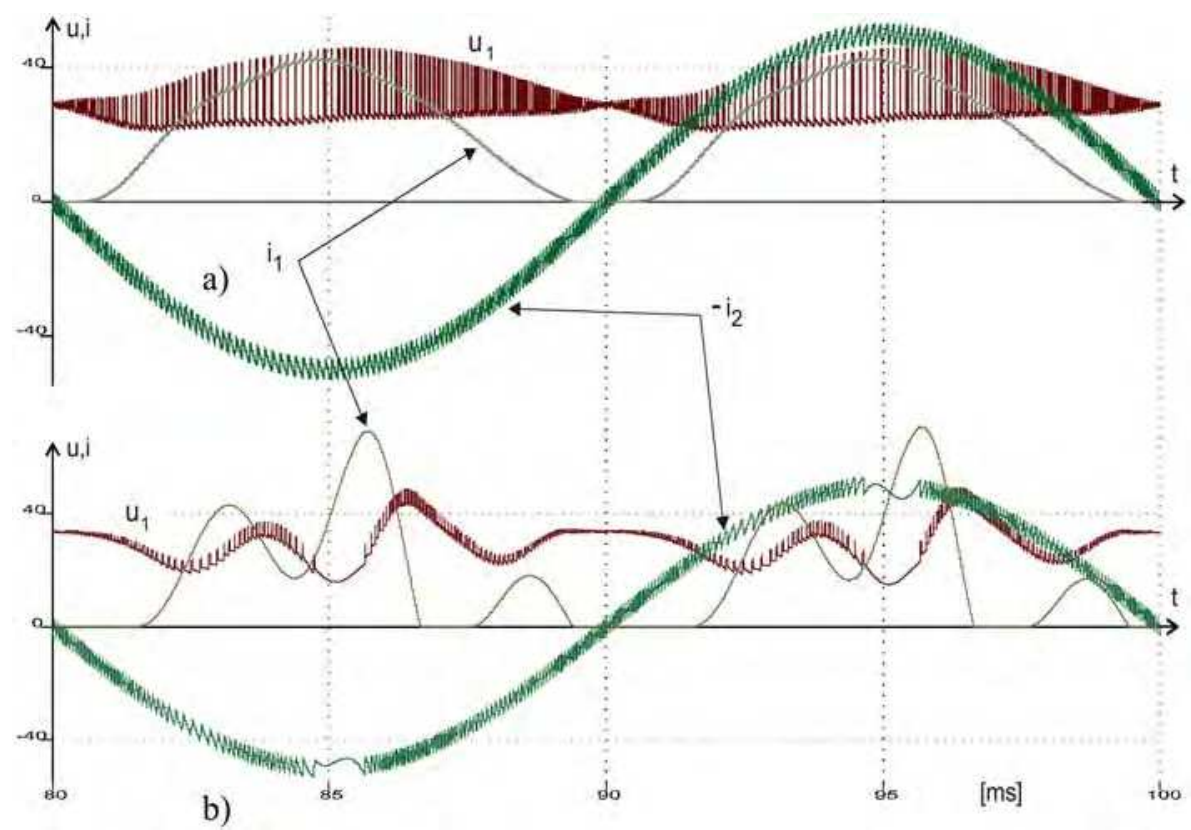

Fig. 8 . The course of filter voltage, $u_{1}$, source, $i_{1}$, and receiver $i_{2}$ currents at filter resistance decreased to the value: a) $R=0,2 \Omega$ and b) $R=0,005 \Omega$

The oscillation can be suppressed by resistor connected into the resonating circuit. In order to minimise power loss, the resistor should be in the branch with capacitor where the current is smaller than in alternator branch.

In order to have full no-oscillating transient in the input circuit with parameters $L_{1}, C$ and $R_{1}+R$, the sum of resistances must fulfil the condition

$$
R_{1}+R>2 \sqrt{\frac{L_{1}}{C}}
$$


which for basic data gives

$$
R_{1}+R>2 \sqrt{\frac{0,175 \cdot 10^{-3}}{10^{-3}}}=0,84 \Omega \text {. }
$$

Figure 8a shows, that satisfying operation is yet at the resistance four times smaller.

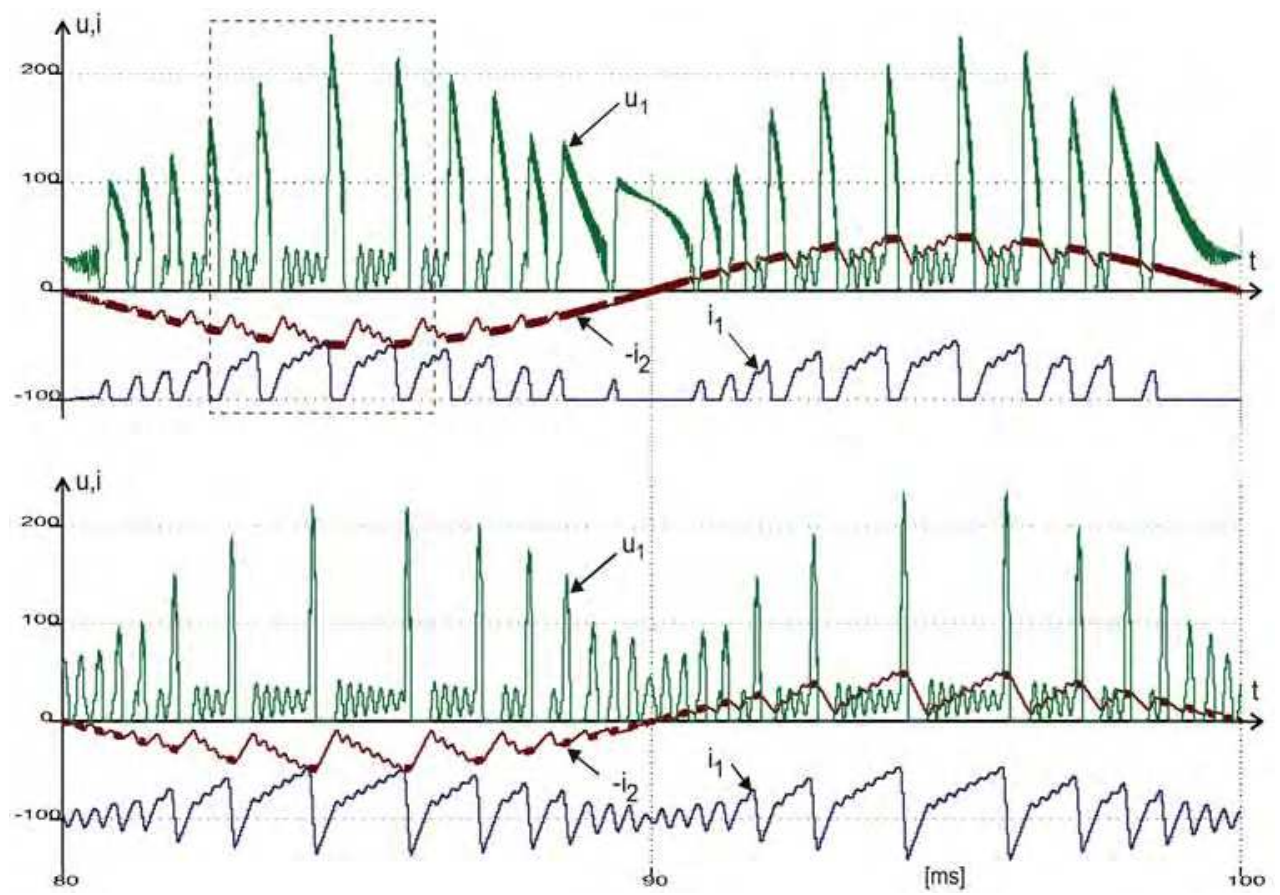

Fig. 9. The course of filter voltage, $u_{1} ; i_{1}$ (with offset equal to $-100 \mathrm{~A}$ ) and $i_{2}$ - currents in the case of non-damped resonance, taking place at zero filter resistance $R$ and decreased filter capacity to the value $C=10 \mu \mathrm{F}$, with (upper figure) and without (lower figure) the diode in the circuits of the source

\subsection{Phenomena at small filter capacitance}

In order to avoid unprofitable low frequency oscillation in the input circuit giving unacceptable disturbance of current forming, the filter capacitance was decreased significantly, at zero filter resistance. It was expected that oscillation of high frequency would not disturb of the current forming in spite of lack of damping the oscillation.

Figure 9 shows the phenomena in the system in the case of zero filter resistance and of distinctly decreased value of the filter capacitance in relation to its basic value (Fig.4a). Due to small value of capacitance, there are changing in turn two states in the system: long duration transistor on state and very high frequency switching state. They can be good analyzed using the time extension of fragment from upper Fig.9 shown in Fig.10.

The long duration on state begins in the time point 1 in Fig.10. In this moment, at zero value of source current $i_{1}$ and at the filter voltage $u_{1}$ equal to or smaller than the actual value of 
EMF $e_{2}$ (Fig.3), the hysteresis comparator switches on the transistor, because the current $i_{2}$ becomes smaller than admissible one (outside the hysteresis band).

In the interval 1-2 the source current $i_{1}$ increases with small slope rate dependent on source inductance $L_{1}$ and charges the filter capacitor. The current $i_{2}$ decreases with the small slope rate dependent on inductance $L_{2}=L_{T}+L_{a}$ as the filter voltage is still smaller than the EMF $e_{2}$. In the moment 2 the filter voltage exceeds $e_{2}$.

In the interval 2-3 the increase of both the currents $i_{1}$ and $i_{2}$ occurs as a result of positive difference between $E_{1}$ and $e_{2}$, with the small slope rate dependent on the sum of inductance $L_{1}+L_{2}$. At the same time the resonance rises up between capacitance and parallel connection of input $L_{1}$ and output $L_{2}$ inductance. There is visible resonance oscillation of the filter voltage $u_{1}$ as well as of the currents $i_{1}$ and $i_{2}$ in Fig. 9 and 10. The parallel resonance has the frequency

$$
f=\frac{1}{2 \pi \sqrt{\frac{L_{1} L_{2}}{L_{1}+L_{2}} C}}
$$

which for $C=10 \mu \mathrm{F}$ and for basic value of inductance $L_{1}$ and $L_{2}$ of the system equals to $5.73 \mathrm{kHz}$. The resonance is insignificant damped due to transformer resistance $R_{2}$. Duration time of the on state is the longer the higher is the actual value of the reference current. In the time point 3 the current $i_{2}$ reaches the upper value of the hysteresis band and in this moment begins the very high frequency switching state.

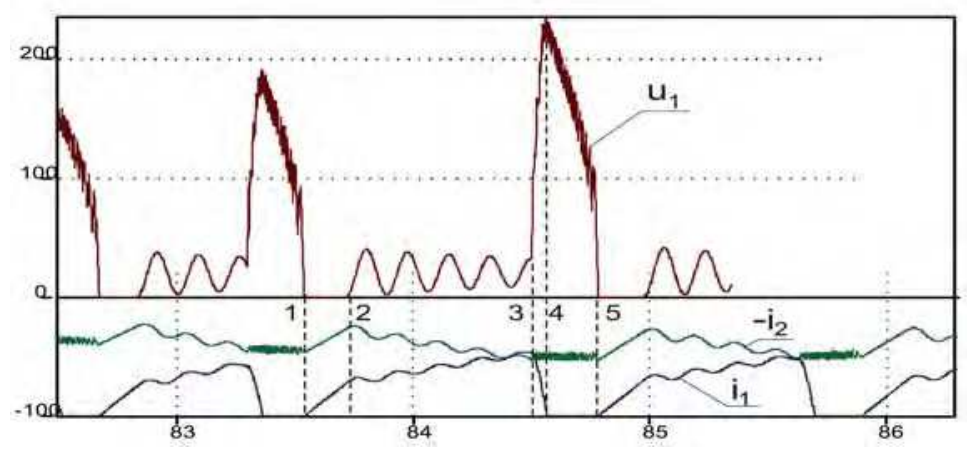

Fig. 10. The case from the upper Fig. 9 with time extension in the region of the current maximum

The process of fast increase of the filter voltage in the period 3-4 is initialized when the transistors switch off first one and the diodes start with conducting. Into the filter two currents are flowing: source current $i_{1}$, forced by inductance $L_{1}$, and current $i_{2}$, forced by inductance $L_{2}$. Both the currents at the beginning of this time period equal approximately to the actual value of the reference current $i_{\text {ref }} n_{T}$. As the capacitance of filter is relatively small its voltage increases rapidly. It leads to the fast decay of the source current $i_{1}$ due to great value of difference $u_{1}-E_{1}$. In result of it the current $i_{1}$ totally disappears. The energy accumulated in inductance $L_{1}$ supplies the filter and causes a high overvoltage on the filter, in spite of discharging the capacitor during next transistor on states. In the time point 4 the voltage $u_{1}$ reaches its maximum value. 
During the interval 4-5 the filter capacitor discharges gradually i.e. with each period of switching the voltage $u_{1}$ becomes lower as the energy flows from the filter to the output circuit. At the end the capacitor is completely discharged. The process repeats, as the end point 5 is a new start point 1 . In the intervals 3-4-5 the filter voltage $u_{1}$ can be many times greater than the receiver EMF $e_{2}$. In the case from Fig. 9 and 10 the voltage exceeds $200 \mathrm{~V}$ at the maximum value of the current. Due to great value of difference $u_{1}-e_{2}$ the very high slope ratio during increasing (transistor on state) and decreasing (transistor off state) of the current $i_{2}$ is in the interval 3-4-5. This is the very high frequency switching state, which is visible as "bold" fragments of the current and voltage shapes in the Fig.9 and 10. Thickness of the current line equals to the width of the hysteresis band. The maximum frequency in the figures exceeds $110 \mathrm{kHz}$.

Neither the long duration on state nor the very high frequency switching one is permissible in the system. The first state gives the long duration error of the current, the second one generates the high switching loss in the transistors and diodes. Then the capacitance of the filter should be sufficiently great in order to eliminate the unprofitable phenomena described above. For the investigated plant the capacitance should be at least several hundred $\mu \mathrm{F}$.

The course of the same phenomena without the diode in the input circuit is shown in the Fig. 9. It relates to the DC generator instead of alternator with diode bridge. The lower figure 9 is similar to upper one. The difference consists in the negative value of the source current $i_{1}$ that is reached in the time when the filter voltage $u_{1}$ is greater than the source EMF $E_{1}$. The increase of the current in the contrary direction takes place at the cost of energy accumulated in the filter capacitor. Therefore, the phase with the very great value of the filter voltage as well as the state of high switching frequency is shorter than in the case with diode. However, the long duration on state is longer as the increase of the current starts from the negative value. It results in the very great error of the current $i_{2}$, which is visible in the lower Fig.9.

It can be stated that the system with source containing diode operates a little better than the system with diode-less source.

\subsection{Current slope rate and switching frequency}

Changing the operation frequency in some range is a disadvantage of converter with direct forming of the current wave. From point of view of loss in the power electronics elements the maximum switching frequency must be limited. The below analysis aims to express the switching frequency as a function of system parameters.

The maximum frequency can be find among two cases of the operation of the system from Fig.2 and 3:

- case 1: the reference current crossing zero is from the negative to positive value (or inversely),

- $\quad$ case 2: the reference current reaches maximum (or minimum) value.

The both cases are illustrated in Fig.11. Duration of increase as well as decrease of the current can be obtained from the geometrical relations. In the Figure 11 the letter $S$ denotes slope of the reference current curve, $S_{1}$ and $S_{3}$ - slopes of the output current $i_{2}$ during its increasing, but $S_{2}$ and $S_{4}$ - during decreasing, for first and second case, respectively.

Duration of the separate phases of the current change, shown in Fig.11, can be expressed in the following way: 
a)

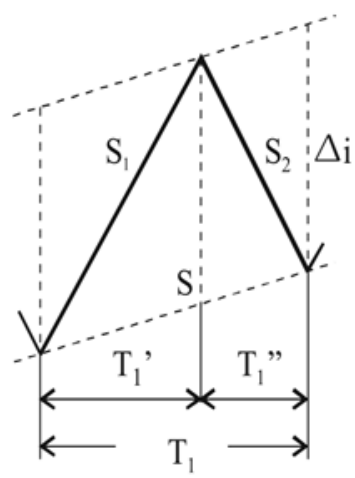

b)

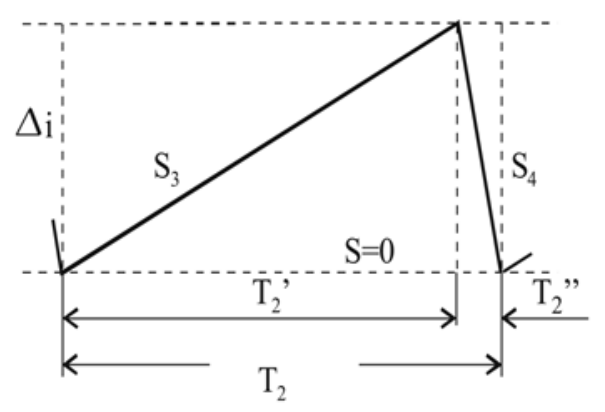

Fig. 11. Fragment of the current course: zero (a-case1) and maximum (b-case 2) neighbourhood

$$
T_{1}^{\prime}=\frac{\Delta i}{S_{1}-S^{\prime}}, \quad T_{1}^{\prime \prime}=\frac{\Delta i}{\left|S_{2}\right|+S}, \quad T_{2}^{\prime}=\frac{\Delta i}{S_{3}}, \quad T_{2}^{\prime \prime}=\frac{\Delta i}{\left|S_{4}\right|} .
$$

Then the periods for case 1 and 2 are:

$$
T_{1}=T_{1}^{\prime}+T_{1}^{\prime \prime}=\frac{S_{1}+\left|S_{2}\right|}{\left(S_{1}-S\right)\left(\left|S_{2}\right|+S\right)} \Delta i
$$

and

$$
T_{2}=T_{2}^{\prime}+T_{2}^{\prime \prime}=\frac{S_{3}+\left|S_{4}\right|}{S_{3} \cdot\left|S_{4}\right|} \Delta i,
$$

respectively. The slope $S$ of the reference current equals to maximum value of its derivative:

$$
S=\omega I_{2 m}
$$

for the case 1 and equals to zero for the case 2 . At this $\omega=2 \pi f$ is the pulsation of the network voltage and reference current.

The slopes of the current $i_{2}$ for the separate phase of its changing equal to the resultant voltage, acting in the circuit, divided by its inductance and are:

$$
S_{1}=\frac{u_{1}}{L_{1}}, \quad S_{2}=\frac{u_{1}}{L_{2}}, \quad S_{3}=\frac{u_{1}-E_{2 m}}{L_{2}}, \quad S_{4}=\frac{u_{1}+E_{2 m}}{L_{2}} .
$$

Inserting the formulas (35) - (39) into (33) - (34) ones, after simply mathematical transformation, the following periods can be obtained:

$$
T_{1}=\frac{2 u_{1} L_{2} \cdot \Delta i}{u_{1}^{2}-\left(\omega L_{2} I_{2 m}\right)^{2}}, \quad T_{2}=\frac{2 u_{1} L_{2} \cdot \Delta i}{u_{1}^{2}-E_{2 m}^{2}} .
$$

The switching frequencies are: 


$$
f_{1}=\frac{u_{1}^{2}-\left(\omega L_{2} I_{2 m}\right)^{2}}{2 u_{1} L_{2} \cdot \Delta i}, \quad f_{2}=\frac{u_{1}^{2}-E_{2 m}^{2}}{2 u_{1} L_{2} \cdot \Delta i} .
$$

The term $\omega L_{2} I_{2 m}$ represents voltage drop on the leakage inductance of the receiver and $E_{2 m}$ represents amplitude of the receiver EMF. As $\omega L_{2} I_{2 m}$ « $E_{2 m}$ the frequency $f_{2}$ « $f_{1}$. It means that the highest frequency is for the case a) from Fig.11 i.e. when the output current and voltage are crossing zero line. It can be also noticed in Fig. 4, 5, 6, 7, 8, 9 and 10. The relation $u_{1}>E_{2 m}$ is a condition of operation of the system. Then the second term in the numerator of (42) can be neglected and the maximum frequency of inverter operation can be written (with accuracy sufficient for practice) in the form:

$$
f_{\max }=\frac{u_{1}}{2 L_{2} \cdot \Delta i} .
$$

For the $u_{1}=E_{1}, L_{2}=L_{T}$ and basic data of the system the maximum frequency is $40,2 \mathrm{kHz}$.

Formula (44) shows the next problem of the small plants with hysteresis forming the current. The grid inductance together with inductance eventual transformer between inverter and grid is small and gives high frequency, unacceptable, even when the hysteresis band is wide. For decrease of frequency the external choke should be added. The value of its inductance must be chosen in compromised way, taking into consideration the loss in the choke and voltage drop (14), which deteriorate the efficiency of the converter.

Formula (44) shows also that high values of filter voltage $u_{1}$ (overvoltage) are unprofitable also from point of view of operation frequency, whose maximum value is proportional to $u_{1}$. Very high frequency can be noticed during time periods with overvoltage registered in Fig.8b, 9 and 10 .

\section{Laboratory plant}

The laboratory converter was built on the base of IGBT module of SKM 75 GB 124 D type with IR2110 gate driver. In the control system the hysteresis comparator LM339 with integrated circuit CD4041 was used. There was network transformer with diode rectifier instead of alternator on the source side.

The capacity of filter was $4,4 \mathrm{mF}$. Filter was without resistance as the source resistance damped sufficiently oscillations in the input circuit. The output transformer had leakage inductance equal to $0,07 \mathrm{mH}$. In order to decrease switching frequency the inductance of $1 \mathrm{mH}$ was serial added. The additional inductor decreased efficiency of the energy transfer to about $50 \%$ at output power about $100 \mathrm{~W}$.

Figure 12 shows operation of the system. The great rate of current slope during diode conducting in the region of maximum $i_{2}$ is visible. Switching frequency changes from about $970 \mathrm{~Hz}$ to about $7500 \mathrm{~Hz}$ when the reference current changes from maximum to zero. Due to great value of inner impedance of source, the filter voltage changes by a few volts according to current pulsation, in spite of great value of filter capacitance.

\section{Cost and reliability oriented design of the converter}

\subsection{The need of compromised optimization of the system}

Preliminary theoretical analysis as well as simulation and laboratory investigations of the inverter (Muszynski \& Pilacinski, 2006; Muszynski \& Pilacinski, 2007; Luczkowski \& 


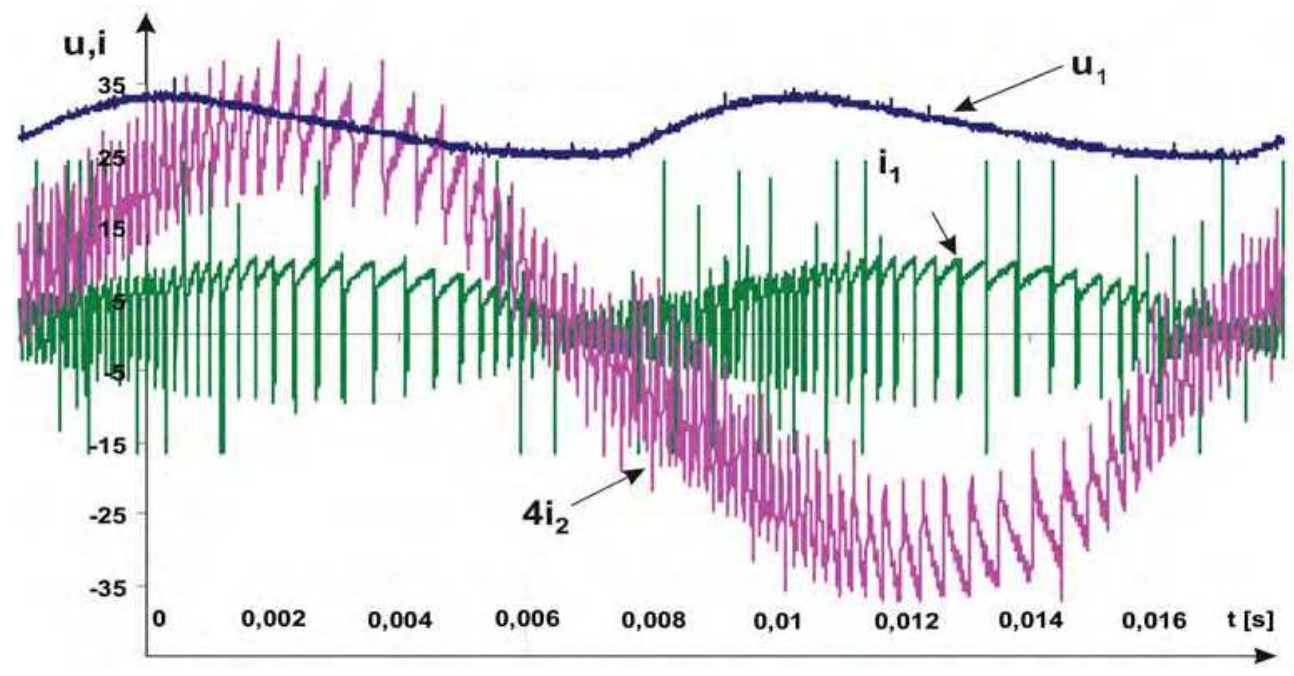

Fig. 12. The course of filter voltage $u_{1}$, current $i_{1}$ of source and current $i_{2}$ of receiver in the laboratory plant at $\mathrm{I}_{2 \mathrm{~m}}=8 \mathrm{~A}$ and $\Delta \mathrm{i}=5 \mathrm{~A}$

Muszynski, 2007) allowed identifying the problems. In the system there is very closed correlation of the circuit and control parameters with the reliability and efficiency of its operation. Every choice of the design parameters has influence on capital cost, on power loss (exploitation effects) and on the level of the reliability. The problem is composed as the system has many design parameters, the partial criteria have different physical nature and their values can be found only for the separate combination of the parameters by means of simulation.

This section presents methodology of designing the converter with consideration of the above mentioned problems.

If the values of the source parameters: EMF $E_{1}$ and inductance $L_{1}$ as well as of the grid and transformer parameters: their short circuit inductance and resistance are given, then at least four other parameters should be chosen during designing. They are the following parameters: filter capacitance $C$ and resistance $R$ in the input circuit, hysteresis band $\Delta i$ of the controller and additional inductance $L_{a}$ in the output circuit. The filter resistance $R$ is needed for damping the resonance oscillation in the input circuit while additional choke $L_{a}$ is necessary in the output circuit for decreasing the current slope rate and frequency of the inverter operation.

These four parameters influence many quantities and indexes of the system. Among them are the over-voltage and voltage class of all elements, frequency and damping decrement of the oscillation in the input circuit, current slope rate and switching frequency of the inverter. Therefore, they influence capital cost, power loss (exploitation effect) as well as reliability of the system.

Some of the requirements are opposite. For instance, introduction of the additional inductance $L_{a}$ is profitable from point of view of the current slope rate and switching frequency. But due to voltage drop on the choke (its inductance and resistance) the voltage adjustment of source (alternator) to the receiver (grid) becomes worse and the additional power is dissipated. 
Due to above feature of the designed system the special compromised its design is proposed.

\subsection{Optimization methodology}

In order to consider during designing the above mentioned requirements of different nature a special generalized optimization criterion

$$
G=p Y=\min
$$

was used where $p$ is the penalty function and $Y$ is the one year cost

$$
Y=C_{C}+C_{L}
$$

having two components: $C_{C}$ equal to the capital cost of the system divided by number of years of the plant operation live and $C_{L}$ equal to the cost of energy lost in the converter per one year of its operation.

The penalty function considers unreliability of the converter and has the form

$$
p=2-H
$$

where $H$ is the two-exponential desirability function (Harrington, 1965) in the form

$$
H=\exp [-\exp (-h)]
$$

The function $H$ (Fig. 13) has many good properties (Harrington, 1965) and is suitable for reliability evaluation. It equals to 1 (practically for $h \geq 5$ ) if the operation of the converter with given combination of parameters is totally reliable (acceptable) and equals to 0 (practically for $h \leq-2$ ) if the operation is totally unreliable (unacceptable).

As a measure of quality of inverter operation (reliability) can be used index

$$
Q=\frac{\Delta i_{r}}{\Delta i_{d}}
$$

where $\Delta i_{d}$ is the desired hysteresis band of the current and $\Delta i_{r}$ is the really reached band. The operation is fully reliable if the controller is able to keep the current in the hysteresis band. For this case $Q \leq Q_{a}$ where $Q_{a}$ is the totally acceptable value of the quality index. Above fully acceptable value $Q_{a}$ begins operation with deteriorated forming the current. User of the plant decides about the value $Q_{a}$ as well as about the value $Q_{u}$ at which the operation is treated as fully acceptable or unacceptable (unreliable).

The quality index $Q$ is transformed into the dimensionless variable $h$ used in (48). The transformation can be linear according to formula

$$
h=a+b Q
$$

where constants $a$ and $b$ are calculated from the conditions: if $Q=Q_{a}$ then $h=5$ and if $Q=Q_{u}$ then $h=-2$, which allow to obtain the characteristic shown in Fig. 13 (Harrington, 1965). As result of it the coefficients are:

$$
a=-\frac{2 Q_{a}+5 Q_{u}}{Q_{a}-Q_{u}}, \quad b=\frac{7}{Q_{a}-Q_{u}} .
$$




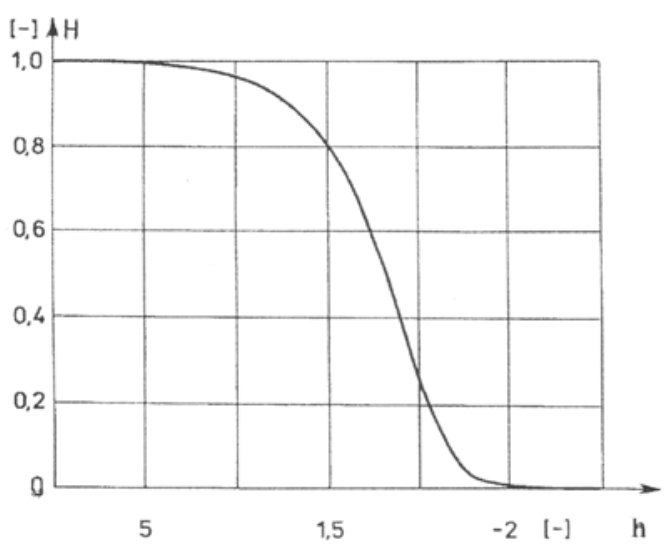

Fig. 13. The desirability function

If for instance the fully reliable value $Q_{a}=0.5$ and the fully unreliable one $Q_{u}=2$ then for this choice the coefficients are: $a=7.333$ and $b=-4.667$.

\subsection{Course of design}

During optimizing the data are exchanged between three blocks in Fig.14.

In the SIMULATION MODEL of the converter for each set of parameters $R, C, \Delta i$ and $L_{a}$ the values of dissipated energy $e$, maximum filter voltage $U_{m}$, maximum frequency $f_{m}$ of transistor switching and effectively reached band $\Delta i_{e}$ of current forming are obtained. These values together with the model parameters $C$ and $L_{a}$ are base for calculating all quantities used in formulas (46) to (50) and finally the generalized optimization criterion G. For

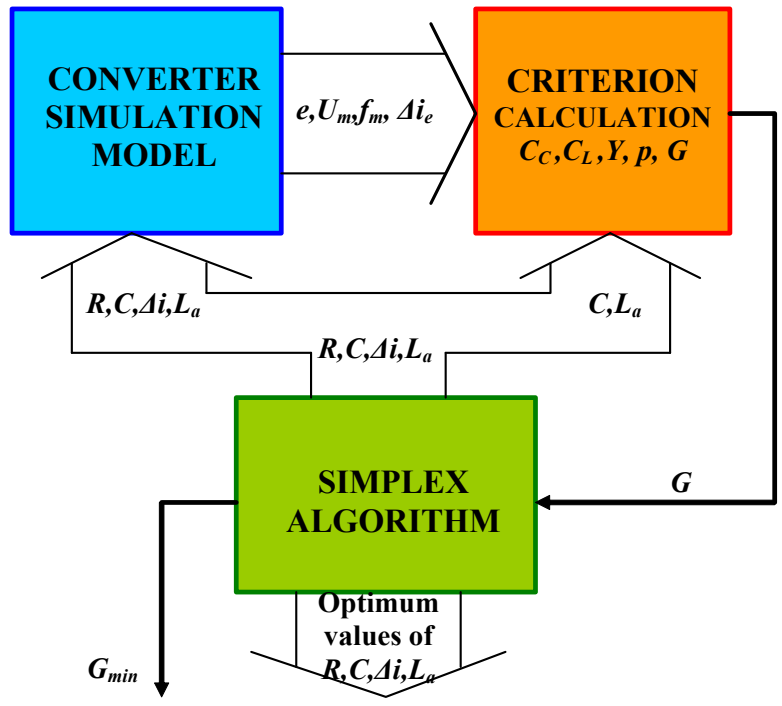

Fig. 14. Block diagram of the optimization 
simulation of the system the TCad Power Electronic Simulation Software (Nieznanski et el., 1996) was used.

The formulas as well as dependencies for obtaining the capital cost $C_{C}$ and the $\operatorname{cost} C_{L}$ of lost energy are programmed into the block CRITERION CALCULATION shown in Fig. 14.

For searching after the optimum solution in sense of (45) the SIMPLEX ALGORITHM of multidimensional optimization (Spendley et al., 1962) was used. It generates the successive sets of the inverter model parameters until the minimum value of the generalized index $G_{\text {min }}$ is reached. The simplex method is proper for optimization using modeling as it gives possibility to reduce notably the needed number of simulations.

The course of optimisation is shown in Fig. 15. For the incoming parameters $R=0.48 \Omega, C=$ $1 \mathrm{mF}, \Delta i=6 \mathrm{~A}$ and $L_{a}=0.08 \mathrm{mH}$ the algorithm has found after 16 steps the outgoing optimum set: $R=0.112 \Omega, C=0.94 \mathrm{mF}, \Delta i=3.49$ A and $L_{a}=0.0882 \mathrm{mH}$. At this the generalized index (45) has been reduced from $75 €$ to $51.8 €$.

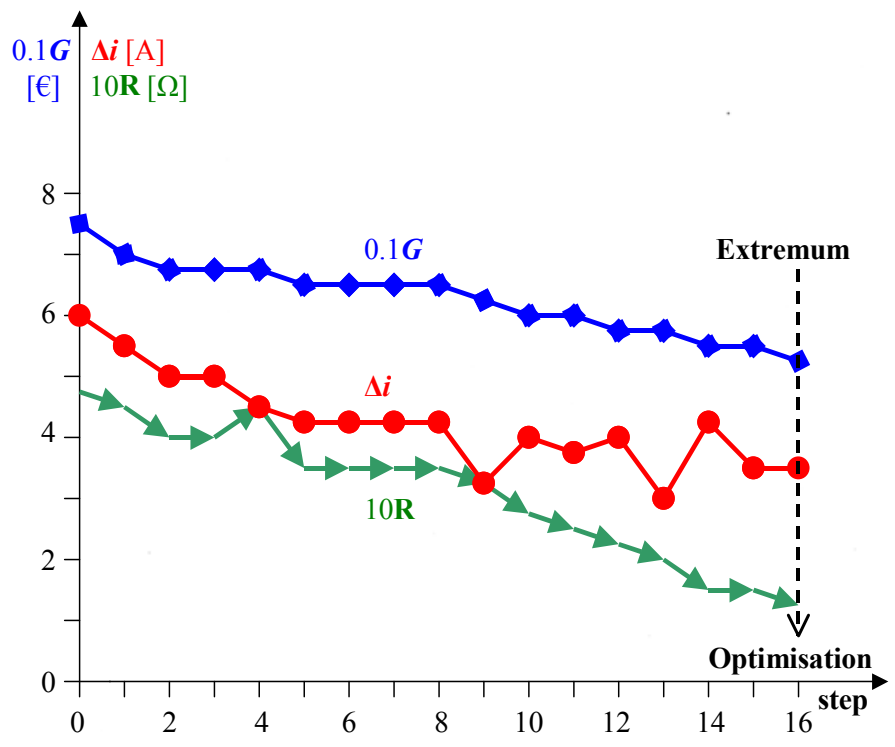

Fig. 15. Course of the values of generalized criterion, current band of the controller and filter resistance during optimization

\section{Conclusions}

The specific proportion of the parameters in the system: Small power source - Inverter with hysteresis controller - Grid causes unprofitable phenomena and difficulties with choice of the filter parameters, hysteresis band and other parameters assuring its good operation.

The theoretical analysis of the phenomena in the circuits of the converter as well as its simulation and laboratory investigations were carried over.

The system needs the great value of filter capacitance and the resistance for damping the low frequency resonance oscillation in the input circuit and the additional inductance for decreasing the current slope rate and switching frequency in the output circuit. 
The design of the system is more difficult than the design of the same structure drive system where the energy flows through the inverter from high power source to the motor.

The task of design of the inverter for energy transfer from small renewable source to common grid should be solved with consideration of power loss, operation reliability and cost of the system. The requirements are bounded here very close one with another and partially are opposed.

The methodology of designing the converter with consideration of the above-mentioned problems was presented.

The solution consists in suitable aggregating the separate requirements in one generalized optimization criterion as well as in using two-exponential desirability function for reliability evaluation and the effective optimization method in order to decrease the simulation number.

The proposed designing gave notable improvement of the inverter properties at small number of optimization steps.

\section{References}

Chikaraishi, H., Hayashi, Y., Sato N. (1990). A variable speed control of the induction generator without speed sensor for wind generation. Trans. IEE Japan. Vol. 110-D. No.6., pp. 664672.

El-Tamaly, A.M., Enjeti, P.N., El-Tamaly, H.H. An improved approach to reduce harmonics in the utility interface of wind, photovoltaic and fuel cell power systems. APEC, Vol. 2.

Harrington, E.C. (1965). The desirability function, Industry quality control, Vol. 21, No 10, pp. $494-498$.

Higuchi, Y., Yamamura, N., Ishida, M., Hori, T. (2000). An improvement of performance for small-scaled wind power generating system with permanent magnet type synchronous generator. Industrial Electronics Society, 2000. 26 th Annual Conference of the IEEE, IECON 2000, Vol. 2., pp. 1037-1043.

Luczkowski, R., Muszynski, R. (2007). Cost and Reliability Oriented Design of the Converter for Small Wind Power Plant, Proceedings of the Colloquium on Reliability in Electromagnetic Systems, May 2007, Paris, France, paper on Conference CD.

Mohan, N., Undeland, T.M., Robbins, W.P. (2003). Power electronics. John Wiley \& Sons, inc.

Muszyński, R., Piłaciński, J.(2006). Small wind power plant with alternator and voltage source inverter. Proc. of the Int. Workshop on Renewable Energy Based Units and Systems, St.Petersburg, June Russia 2006.

Muszynski, R.., Pilacinski, J. (2007). Investigation of the inverter for energy transfer from small renewable source to common grid, Proceedings of the $16^{\text {th }}$ Int. Conference on Electrical Drives and Power Electronics, The High Tatras, September 2007, Slovakia, paper on Conference CD.

Nieznanski, J., Iwan, K., Szczęsny, R., Ronkowski, M. (1996). Tcad for Windows. High Performance Power Electronic Simulation Software, SOFTECH, Gdansk.

Piggott H. (2005). How to build wind turbine. The axial flux windmill plans, Scoraig Wind Electric, UK.

Spendley W., Hext, G.R., Himsworth, F.R. (1962). The sequential application of simplex designs in optimisations - an evolutionary operations, Technometrics, Vol.4, pp. 441-448.

Tyc, M. (2003). The physical model of co-operation of small wind plant with the 1-phase network. The MSc work (consultant R. Muszyński), Poznań University of Technology, Institute of Electrical Engineering and Electronics, Poznan 2003 (in polish). 


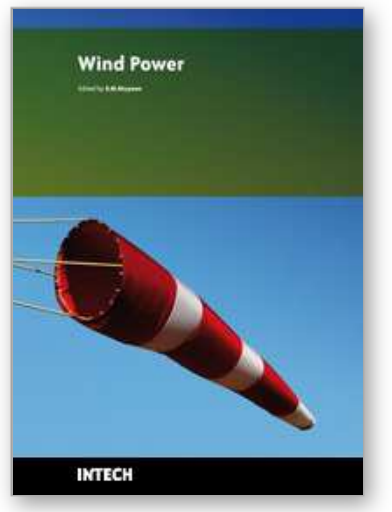

\author{
Wind Power \\ Edited by S M Muyeen
}

ISBN 978-953-7619-81-7

Hard cover, 558 pages

Publisher InTech

Published online 01, June, 2010

Published in print edition June, 2010

This book is the result of inspirations and contributions from many researchers of different fields. A wide verity of research results are merged together to make this book useful for students and researchers who will take contribution for further development of the existing technology. I hope you will enjoy the book, so that my effort to bringing it together for you will be successful. In my capacity, as the Editor of this book, I would like to thanks and appreciate the chapter authors, who ensured the quality of the material as well as submitting their best works. Most of the results presented in to the book have already been published on international journals and appreciated in many international conferences.

\title{
How to reference
}

In order to correctly reference this scholarly work, feel free to copy and paste the following:

Romuald Luczkowski and Roman Muszynski (2010). Analysis and Investigation of the Inverter for Energy Transfer from Small Wind Power Plant to Common Grid, Wind Power, S M Muyeen (Ed.), ISBN: 978-9537619-81-7, InTech, Available from: http://www.intechopen.com/books/wind-power/analysis-and-investigationof-the-inverter-for-energy-transfer-from-small-wind-power-plant-to-common

\section{INTECH}

open science | open minds

\section{InTech Europe}

University Campus STeP Ri Slavka Krautzeka 83/A 51000 Rijeka, Croatia

Phone: +385 (51) 770447

Fax: +385 (51) 686166 www.intechopen.com

\section{InTech China}

Unit 405, Office Block, Hotel Equatorial Shanghai No.65, Yan An Road (West), Shanghai, 200040, China 中国上海市延安西路65号上海国际贵都大饭店办公楼405单元 Phone: +86-21-62489820

Fax: +86-21-62489821 
(C) 2010 The Author(s). Licensee IntechOpen. This chapter is distributed under the terms of the Creative Commons Attribution-NonCommercialShareAlike-3.0 License, which permits use, distribution and reproduction for non-commercial purposes, provided the original is properly cited and derivative works building on this content are distributed under the same license. 